\title{
The size of the population potentially in need of palliative care in Germany - an estimation based on death registration data
}

Nadine Scholten ${ }^{*}$, Anna Lena Günther, Holger Pfaff and Ute Karbach

\begin{abstract}
Background: No data exist on the size of the population potentially in need of palliative care in Germany. The aim of this study is to estimate the size of the German population that may benefit from palliative care.

Method: Based on existing population-based methods (Rosenwax and Murtagh), German death registration data were analyzed and contrasted with international results. The data include all death cases in 2013 in Germany.

Results: According to the method Rosenwax defined, between $40.7 \%$ (minimal estimate) and $96.1 \%$ (maximal estimate) of death cases could benefit from palliative care. The estimation, based on Murtagh's refined method, results in $78.0 \%$ of death cases potentially being eligible for palliative care. The percentage of potential palliative care candidates is conditioned by age. Based on the Murtagh Method, in the age category between 30 and 39 years, a potential demand for palliative care can be found for $40.4 \%$ percent of all deaths occurring in this age category, with this number increasing to $80.3 \%$ in the age bracket of 80 years and over.
\end{abstract}

Conclusion: An estimation of the size of the population in need is essential for healthcare planning. Therefore, our data serve as a guide and starting point for further research.

Keywords: Palliative care, Palliative need, Population-based estimate, End-of-life care, Death registration data

\section{Background}

In an aging society, the burden of dealing with cancer and other chronic life-threatening diseases increases. According to the WHO (World Health Organization), the aim of palliative care, as an interdisciplinary profession, is to provide symptom control and support for affected patients and their families who are facing a terminal illness [1]. Palliative care has been established to focus on relieving pain in cancer patients [2]. Until now, palliative care has been centered around the treatment of cancer patients [3, 4], although there has been increasing research showing the demand for palliative care in chronic non-cancer patients with a terminal diagnosis [5-7]. The

\footnotetext{
* Correspondence: nadine.scholten@uk-koeln.de

IMVR - Institute for Medical Sociology, Health Services Research and

Rehabilitation Science, University of Cologne, Cologne, Germany
}

need for palliative care can be defined in many ways [8], including using Bradshaw's categories (felt, expressed, normative, and comparative needs) [9]. The ability of patients to benefit from palliative care (normative perspective) seems to be a useful approach for defining need in this context [10]. In Germany, the majority of patients in palliative care services are cancer patients [3]. Because terminally ill patients with cancer, as well as those with non-cancer diagnoses, benefit from palliative care, it is important to enable access for all patients that could benefit from palliative care [5-7]. In order to provide the needed care and to make adequate palliative care services available, it is necessary to have data on the actual need for care. Regarding palliative care, there is a distinction to be made between primary palliative care provided by general practitioners, for example, or specialized palliative care supplied by physicians specializing 
in palliative care, for example in hospitals or ambulatory teams [11].

\section{Palliative care in Germany}

Palliative care is an emerging medical field in Germany, but compared to Great Britain, which is considered the pioneer in the development of palliative care [12], more action needs to be taken to catch up, even though Germany is improving [13]. According to the Quality of Death Index 2015, published by the Economist, Germany ranks number 7 behind Great Britain (No. 1), Australia (No. 2) and New Zealand (No. 3) [14]. Palliative care has been stated to be a human right [15]. With the healthcare reforms in 2007 in Germany, every patient in need of specialized palliative care should be able to receive that care [16].

\section{Population based methods to estimate need for palliative care}

Based on Western Australian death record data, Rosenwax et al. [17] developed a method in 2005 to estimate the minimal, mid-range, and maximal numbers of people that could potentially benefit from palliative care during their last year of life based on administrative data. For the minimal estimate, 10 medical conditions (neoplasms, heart failure, renal failure, chronic obstructive pulmonary disease, Alzheimer's disease, liver failure, Parkinson's disease, motor neuron disease, Huntington's disease, and HIV/AIDS) were determined using focus groups, interviews, and literature reviews, identifying affected patients who could benefit from palliative care before death. The codes for these medical conditions are used to identify the population of individuals that are potentially in need for palliative care within death registration data. In other words, everybody who died because of one of the defined conditions is classified as a palliative care candidate. The mid-range estimate resulted from matching death certificate data with hospital data, including certain hospital admissions during the last year of life. The maximal estimate is based on all deaths, excluding death during pregnancy, childbirth, or the puerperium, originating during the perinatal period, or resulting from external causes, like injury or poisoning, as palliative care does not seem appropriate in these populations [17].

Murtagh et al. refined this method in 2014 because, according to the authors, the minimal method Rosenwax et al. described underestimates the number of people who may need palliative care. Additional diagnosis codes were added and others were eliminated to account for changes in diagnostic and treatment practices. Benign neoplasms were excluded, and chronic heart diseases, hypertensive and ischemic heart diseases, and cerebrovascular diseases were included. The categories of renal and liver failures were extended to include chronic renal and liver failures as well. Other chronic, respiratory, and neurodegenerative diseases were included, like multiple sclerosis and progressive supranuclear palsy. Additionally, all types of dementia and Alzheimer's diseases were added to the Rosenwax classification [10].

The results of the Rosenwax method were contrasted with the Murtagh method, based on deaths in England occurring between 2006 and 2008. The comparison showed an increase from 37 to $63 \%$ for all cases of death that were potentially in need of palliative care [10].

\section{Objectives}

In Germany, no comprehensive population study exists on the demand for palliative care and its need within the population. Therefore, the aim of our study was to obtain a range of estimations to get a sense of how many people may be in need of palliative care. These data can be seen as baseline data for health system planning and are the basis for further discussion about palliative care needs in cancer and non-cancer patients. The current study was based on Murtagh et al. and Rosenwax et al.'s methods. The findings were then contrasted with international data.

\section{Method}

This study was based on three (Rosenwax's minimal and maximal estimations and Murtagh's adapted version) of the existing methods to estimate the number of people who are possibly in need of palliative care. Data on the number and causes of deaths were derived from death registration data. This population-based database, published annually by the Federal Bureau of Statistics, is a complete inventory count of all cases of death in Germany. It contains information on the cause of death, as well as sex and age. The cause of death is coded using the ICD-10-WHO code, making international comparisons possible [18]. Based on the death certificate, qualified coders identify the underlying cause of death and document the relevant code. According to the $\mathrm{WHO}$, the mortality statistics contain the main underlying cause of death (monocausal) [19]. The mortality statistics can be seen as secondary statistics and can be used for epidemiological research, regional comparisons, and health services research. As it is not possible in Germany to match the mortality statistics with hospital admission data, our analyses were restricted to the Rosenwax minimal and maximal estimate method [17] and the Murtagh method [10], an adaption of the Rosenwax minimal estimate method, performed by Murtagh et al. The database used for our calculation is the German Mortality Statistics 2013, as it is the latest data year available at this point.

To transfer the Rosenwax (Australia) and Murtagh (Great Britain) methods to German data, some adaptions had to be made to convert the defined codes to ICD-10- 
WHO year 2013 codes. This has been necessary, as the ICD-Codes between countries and years can differ (See Table 1 for the utilized ICD-10-WHO year 2013 codes). To apply the Rosenwax method to German data, the ICD-10-AM codes had to be converted to ICD-10WHO codes. The ICD-codes our analyses are based on can be found in Table 1 .

The maximal estimate codes, defined by Rosenwax et al. and used by Murtagh et al., were converted into ICD$10-$ WHO codes that did not need any adaptions. The maximal estimate includes all deaths except those due to one of the following conditions:

- Pregnancy, childbirth, or puerperium (ICD-10-WHO codes O00-O99)

- Those originating during the perinatal period (ICD-10-WHO codes P00-P96)

- Injury, poisoning, and other similar causes (ICD-10WHO codes S00-T98)

- External causes (ICD-10-WHO codes V01-Y98)
The database provided by the Federal Bureau of Statistics contains no data on the personal level, and is therefore open to the public. Thus, obtaining ethical approval is not necessary.

\section{Results}

A total of 893,825 people died in Germany in 2013, with the majority dying from diseases of the circulatory system (39.7\%), followed by neoplasms (25.0\%). According to the Rosenwax method, a total of $363,689(40.7 \%)$ deaths were potentially in need of palliative care in Germany in 2013. Neoplasms accounted for $63.5 \%$ of all deaths with regard to all potential palliative care candidates identified using the Rosenwax method.

Using the Murtagh method, the number of people who could benefit from palliative care was 697,281 (78.0 \%), which is almost twice as many people compared to the Rosenwax method. The largest deviation between the Murtagh and Rosenwax methods could be found within the category "heart," where almost $60.0 \%$

Table 1 Comparison of the Rosenwax and Murtagh methods for estimating the need for palliative care

\begin{tabular}{|c|c|c|c|c|c|c|}
\hline \multicolumn{2}{|l|}{ Rosenwax Method } & \multirow{2}{*}{$\begin{array}{l}n \\
230.840(25.82 \%)\end{array}$} & \multicolumn{2}{|l|}{ Murtagh Method } & \multirow{2}{*}{$\begin{array}{l}n \\
223.842(25.04 \%)\end{array}$} & \multirow{2}{*}{$\begin{array}{l}\text { Difference } \\
6.998\end{array}$} \\
\hline Neoplasm & C00-D48 & & $\begin{array}{l}\text { Malignant } \\
\text { neoplasm }\end{array}$ & C00-C97 & & \\
\hline & & & Cancer (breast) & C50 & 18.009 (2.01\%) & \\
\hline & & & Cancer (colorectal) & $\mathrm{C} 18-\mathrm{C} 21$ & $25.693(2.87 \%)$ & \\
\hline & & & Cancer (lung) & C30-C39 & 46.896 (5.25 \%) & \\
\hline & & & Cancer (prostate) & C61 & 13.408 (1.5 \%) & \\
\hline & & & Cancer (other) & & 119.836 (13.41 \%) & \\
\hline \multirow[t]{2}{*}{ Heart failure } & $\begin{array}{l}\mid 110,1119 \\
1500, \mid 1501,1509 \\
1130,1132\end{array}$ & 74.704 (8.36 \%) & $\begin{array}{l}\text { Heart disease } \\
\text { (chronic) }\end{array}$ & $100-152$ & 274.821 (30.75 \%) & 258.673 \\
\hline & & & $\begin{array}{l}\text { Cerebrovascular } \\
\text { disease (stroke) }\end{array}$ & 160-169 & 58.556 (6.55 \%) & \\
\hline Renal failure & $\begin{array}{l}\text { N10, N11, N18, } \\
\text { N120, N131, N132 }\end{array}$ & 8.588 (0.96 \%) & $\begin{array}{l}\text { Renal disease } \\
\text { (chronic renal } \\
\text { failure) }\end{array}$ & $\begin{array}{l}\text { N17, N18, N28, } \\
|12,| 13\end{array}$ & $18.817(2.11 \%)$ & 10.229 \\
\hline Liver failure & $\begin{array}{l}\text { K704, K711, K721, } \\
\text { K729 }\end{array}$ & $493(0.06 \%)$ & Liver disease & K70-K77 & $15.255(1.71 \%)$ & 14.762 \\
\hline $\begin{array}{l}\text { Chronic obstructive } \\
\text { pulmonary disease }\end{array}$ & $\begin{array}{l}J 40, J 410, J 411 \\
J 418, J 42,430-J 449\end{array}$ & 31.570 (3.53 \%) & Respiratory disease & $\begin{array}{l}J 06-J 18, J 20-J 22, \\
J 40-J 47 \& \text { J96 }\end{array}$ & 55.557 (6.22 \%) & 23.987 \\
\hline $\begin{array}{l}\text { Neurodegenerative } \\
\text { disease }\end{array}$ & G10, G122, G20 & $10.841(1.21 \%)$ & $\begin{array}{l}\text { Neurodegenerative } \\
\text { disease }\end{array}$ & $\begin{array}{l}\text { G10, G20, G35, } \\
\text { G122, G903, G231 }\end{array}$ & 12.349 (1.38 \%) & 1.508 \\
\hline Huntington's disease & G10 & $332(0.04 \%)$ & & & & \\
\hline Motor neuron disease & G122 & 1.878 (0.21 \%) & & & & \\
\hline Parkinson's disease & $\mathrm{G} 20$ & 8.631 (0.97 \%) & & & & \\
\hline Alzheimer's disease & $\begin{array}{l}\text { G300, G301, G308, } \\
\text { G309 }\end{array}$ & $6.252(0.70 \%)$ & $\begin{array}{l}\text { Dementia, } \\
\text { Alzheimer's, senility }\end{array}$ & F01, F03, G30, R54 & $37.683(4.22 \%)$ & 31.431 \\
\hline HIV/AIDS & B20-B24 & $401(0.04 \%)$ & HIV/AIDS & B20-B24 & 401 (0.045 \%) & 0 \\
\hline $\begin{array}{l}\text { Total deaths from } \\
\text { these conditions }\end{array}$ & & $\begin{array}{l}363.689 \mathbf{4 0 . 7} \% \text { of all } \\
\text { deaths }(n=893.825)\end{array}$ & $\begin{array}{l}\text { Total deaths from } \\
\text { these conditions }\end{array}$ & & $\begin{array}{l}697.28178 .0 \% \text { of all } \\
\text { deaths }(n=893.825)\end{array}$ & \\
\hline
\end{tabular}

A transformation of the Rosenwax ICD-10-AM to ICD-10-WHO codes was not possible for codes I111, N102, and N112. Hence, we replaced these codes with codes I11.0, I11.9, N10, N11.1, N11.8, and N11.9 ICD-10-WHO 
of the deviation can be explained. The proportion of patients in need of palliative care because of a heart disease rises from $8.4 \%$ (Rosenwax) to 30.8 \% (Murtagh), making heart disease the most frequent underlying cause for the potential demand of palliative care.

According to the maximal estimate originally developed by Rosenwax et al. [17], a total of 858,546 people, accounting for $96.1 \%$ of all deaths, could be potential palliative care patients. The Rosenwax minimal method identifies $40.7 \%$ of all deaths eligible for palliative care, and Murtagh's adaption of the Rosenwax method for a minimal estimate expands the demand to $78.0 \%$ of all deaths (Fig. 1).

To give further insight into age-related differences regarding the demand for palliative care, we performed an additional analysis accounting for the factor "age". We estimated the demand for palliative care within the different age groups with the Murtagh method and the maximal estimate (Rosenwax method). The results showed that, according to Murtagh, the demand in children aged 0 to 9 years lies at $9.9 \%$ of all deaths occurring in this age category. The percentage of potential palliative care candidates rises to $40.4 \%$ in the age category between 30 and 39 years and keeps increasing to $80.3 \%$ in people aged 80 and over. Similar results, but on a higher level (10-15 percentage points higher than the Murtagh method), can be found for the maximal estimate, with the biggest discrepancies within the age category 0 to 9 years (almost 25 percentage points higher). The increase of potential palliative care needs with older age is caused by a change of the cause of death from more external causes of death, like accidents, to more cases of death caused by diseases, potentially creating a need for palliative care (e.g. cancer and vascular diseases) (Figs. 2 and 3).

\section{Discussion}

Comparing the need for palliative care in the German population, we can find similar results to those from
Western Australia and Great Britain. By using the Rosenwax method, we identified $40.7 \%$ of all deaths occurring in Germany in 2013 as being caused by conditions Rosenwax et al. defined. Murtagh et al. found similar results for England, indicating that $37 \%$ of all deaths may have been preceded by a need for palliative care [10]. The percentage, according Rosenwax et al.'s minimal estimate, is $50 \%$ of all deaths for Western Australia [17]. Worldwide, the percentage ranges from between $25 \%$ (Mexico) and $49 \%$ (Netherlands), whereas in Western Europe (excluding Germany), the lowest rate lies at $41 \%$ (Wales) [20]. Murtagh et al. adapted Rosenwax's minimal estimate method by adding extra conditions that, in the opinion of questioned experts, palliative care seemed to be appropriate for. Other conditions where palliative care did not seem appropriate were deleted [10]. For England, an estimated $63 \%$ of all deaths fall in the categories defined by Murtagh et al [10]. By using the Murtagh method, $78 \%$ of all deaths in Germany would potentially be in need of palliative care. This percentage is substantially higher than the findings from England. Here, the highest discrepancy was found with regard to coded deaths due to heart disease. In Germany in 2013, the underlying cause of death was a heart disease in approximately $31 \%$ of all cases, whereas in England, only about $12 \%$ of all deaths were caused by heart disease [10]. Comparing our data to mortality data from the WHO in 2013, Germany has an age-standardized death rate for diseases of the circulatory system of 145.5 per 100,000 , compared to the United Kingdom (104.3) or the United States (131.2) [21]. In addition to the higher mean age of the German population, differences in the population's specific death rate for the circulatory system could partly explain the discrepancies found. Comparing the need for palliative care with data from Australia [17] and Great Britain [10] for people dying at an age over 65, Germany seems to have the highest demand for palliative care

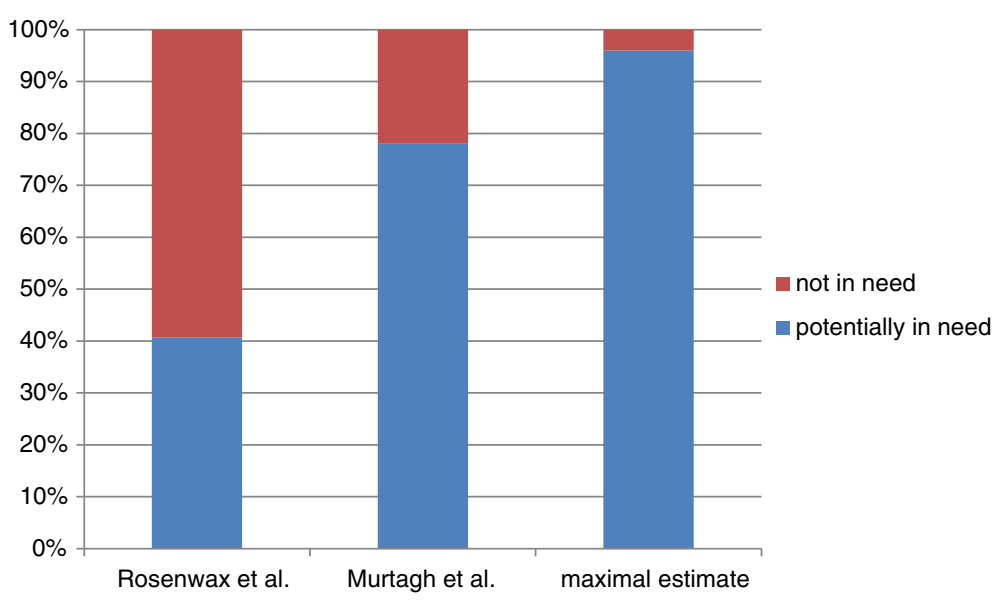

Fig. 1 Percentage of death cases classified as potentially in need vs. not in need 


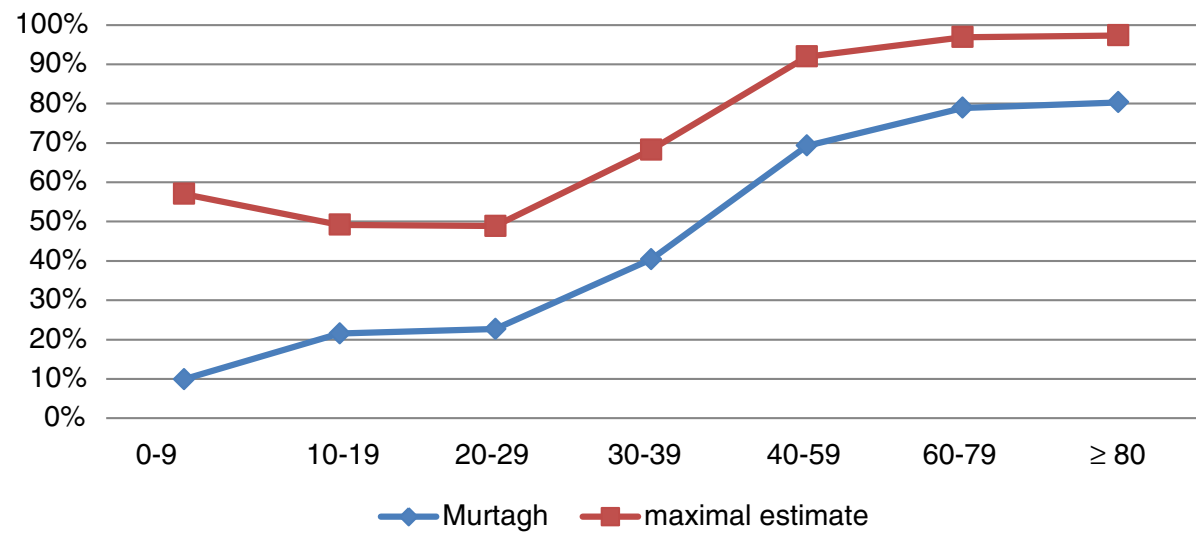

Fig. 2 Demand for palliative care by age group

in this age group. In Germany, people dying over the age of 65 account for $84.4 \%$ of all death cases, while in Western Australia only $77.0 \%$ of deaths occur over the age of 65 [17].

With a median age of 46.5 years, Germany is the second oldest nation worldwide, with only Japan having a higher median age [22]. With older age, more and more people die from conditions potentially generating a need for palliative care; therefore, in an aging society, a higher demand for palliative care can be assumed.

The need for palliative care has to be seen alongside with the supply of palliative care in the corresponding country. Prior estimations for Germany stated a demand of 80 to 100 hospital beds (hospice and palliative care) per million inhabitants [13]. Inpatient palliative care is one of many ways to deliver palliative care. As the majority of Germans with terminal diseases wish to die at home [23], there is a need to provide palliative care at home. Because patients benefit from early access to palliative care $[24,25]$ and costs can be cut [26], it is important to implement palliative care early in care trajectories, making the demand for palliative care even higher, as the utilization is not restricted to the time shortly before death. Until now, it was mainly patients with cancer who received palliative care in Germany [27], although many terminally ill patients with a non-cancer disease benefit from palliative care as well [5-7].

\section{Limitations}

The Rosenwax and the Murtagh methods are based on death certificate data. The limitations of the Rosenwax and Murtagh methods have been widely discussed [10, 17, 28]; therefore, at this point, we only want to give a short summary of the main limitations. Both measures are based on death certificate data, whereby some diagnoses (e.g. dementia, Parkinson's or renal diseases) may be under-recorded [10], especially when only the underlying cause of death has been documented. Population-based measures, like the ones Rosenwax and Murtagh utilized are conditionbased and are therefore not able to measure the patients' actual needs for palliative care [10, 17, 28], as need is determined by many factors, in addition to the diagnosis alone $[29,30]$. Severity, in addition to illness trajectories, is not accounted for. This may be especially relevant when it comes to severely ill children and adolescents, where

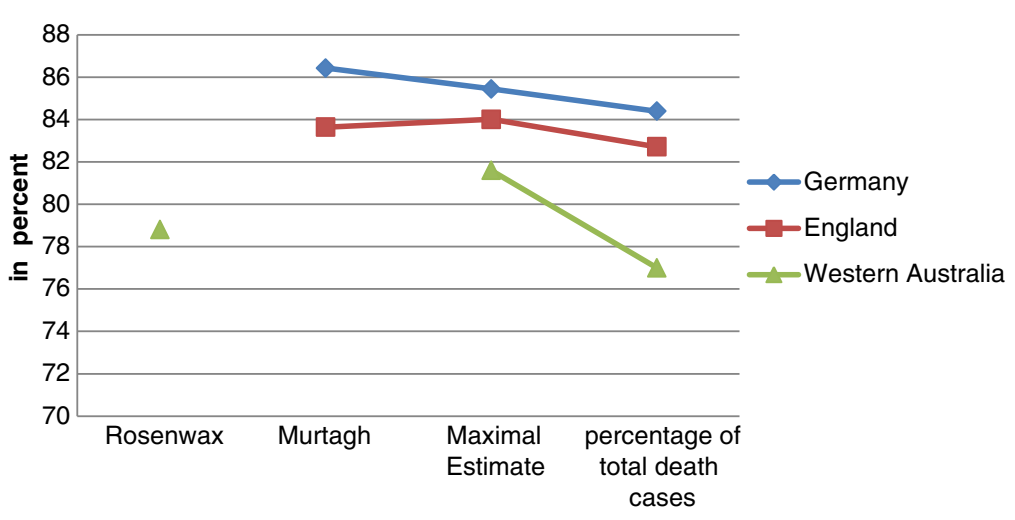

Fig. 3 Demand for palliative care in death cases > 65 years of age incomparison to Rosenwax and Murtagh 
there can be a possible demand for palliative care over the course of years [10]. These estimations on a population basis can be seen as a low-cost, pragmatic approach for policy and resource allocation, including a certain degree of misclassification [28].

If further data are available, other methods do exist to perform estimations on the realistic need for palliative care in a population. For example, Gómez-Batiste et al. performed an analysis based on the prevalence of symptoms, chronic conditions, and structural information regarding the rate of home residences [31]. Rosenwax et al. performed further estimations by linking hospital admission data to get a mid-range estimate, including all deaths with a hospitalization record one year prior to death because of the same condition that was documented in the death certificate [17]. As it is not possible to link further data to this nationwide German dataset, our results serve as a rough estimation of the palliative care needs in Germany. Further studies, based on health insurance companies' data, could provide further information, although these results can lack generalizability. Our study is based on data of one single year (2013). Further findings can be obtained using a longitudinal analysis and expansion of the databases to more than one analyzed year.

\section{Conclusion}

These figures (i.e. the amount of non-cancer patients who could benefit from palliative care) can provide clues to guide the future planning of palliative care service delivery for patients with non-cancer diseases - particularly since there is evidence of shortcomings in the provision of palliative care for non-cancer patients in Germany [7].

From 2009 to 2050, the number of deaths in Germany is expected to rise by $25 \%$, with a total increase of death cases and more people dying at an advanced age. With a higher need for palliative care at an older age, palliative care becomes more and more important [32].

After all, "the demand for palliative care" cannot be seen as a given fact, but is partly subject to continuously evolving ideas of what is appropriate care, economic resources and resource allocation. The estimates performed can serve as a rough approximation, highlighting the immense need for palliative care in an aging society like Germany.

\section{Competing interests}

The authors declare that they have no conflicts of interest. This research has not been funded by any funding agency in the public, commercial, or not-for-profit sectors.

\section{Authors' contribution}

SN and UK designed the study, with HP's participation. SN and ALG carried out the data analysis and drafted the manuscript. All authors read and approved the final manuscript.
Received: 18 November 2015 Accepted: 23 February 2016

Published online: 08 March 2016

\section{References}

1. World Health Organization: Definition of palliative care [http://www.who.int/ cancer/palliative/definition/en/. Accessed 1 Feb 2016.].

2. Sepúlveda C, Marlin A, Yoshida T, Ullrich A. Palliative care: the World Health Organization's global perspective. J Pain Symptom Manage. 2002;24:91-6.

3. Hess S, Stiel S, Hofmann S, Klein C, Lindena G, Ostgathe C. Trends in specialized palliative care for non-cancer patients in Germany - Data from the National Hospice and Palliative Care Evaluation (HOPE). Eur J Intern Med. 2014;25:187-92.

4. Korte-Verhoef D, Maria C, Pasman H, Roeline W, Schweitzer, Bart PM, Francke AL, Onwuteaka-Philipsen BD, Deliens L. Reasons for hospitalisation at the end of life: differences between cancer and non-cancer patients. Support Care Cancer. 2014;22:645-52.

5. Solano JP, Gomes B, Higginson IJ. A comparison of symptom prevalence in far advanced cancer, AIDS, heart disease, chronic obstructive pulmonary disease and renal disease. J Pain Symptom Manage. 2006;31:58-69.

6. Moens K, Higginson IJ, Harding R. Are there differences in the prevalence of palliative care-related problems in people living with advanced cancer and eight non-cancer conditions? A systematic review. J Pain Symptom Manage. 2014;48:660-77.

7. Stiel S, Heckel M, Seifert A, Frauendorf T, Hanke RM, Ostgathe C. Comparison of terminally ill cancer- vs. non-cancer patients in specialized palliative home care in Germany - a single service analysis. BMC Palliat Care. 2015;14:271

8. Asadi-Lari M, Packham C, Gray D. Need for redefining needs. Health Qual Life Outcomes. 2003;1:34.

9. Bradshaw J. A taxonomy of social need. In: Bradshaw J, Cookson R, Sainsbury R, Glendinning C, editors. Jonathan Bradshaw on social policy. Selected writings 1972-2011. York: University of York; 2013. p. 1-11.

10. Murtagh, Fliss EM, Bausewein C, Verne J, Groeneveld El, Kaloki YE, Higginson IJ. How many people need palliative care? A study developing and comparing methods for population-based estimates. Palliat Med. 2014;28: 49-58.

11. Radbruch L, Payne S. Standards und Richtlinien für Hospiz- und Palliativversorgung in Europa. Teil 2. Palliativmedizin. 2011:12:260-70.

12. Behmann M, Lückmann SL, Schneider N. Palliative care in Germany from a public health perspective: Qualitative expert interviews. BMC Res Notes. 2009;2:116.

13. Radbruch L, Bausewein C, Simon S, Sipp W, Wodarg W, Jünger S. Europäische Empfehlungen zur Palliativversorgung und Hospizarbeit und ihre Umsetzung in Deutschland. Palliativmedizin. 2011;12:177-85.

14. The 2015 Quality of Death Index: Ranking palliative care across the world; 2015.http://www.economistinsights.com/healthcare/analysis/quality-deathindex-2015.

15. Brennan F. Palliative care as an international human right. J Pain Symptom Manage. 2007;33:494-9.

16. Schneider N. Die neue spezialisierte ambulante Palliativversorgung - ein Positionspapier. Z Allg Med. 2008;84:232-5.

17. Rosenwax LK, McNamara B, Blackmore AM, Holman CDJ. Estimating the size of a potential palliative care population. Palliat Med. 2005;19:556-62.

18. Statistisches Bundesamt: Todesursachenstatistik [https://www.destatis.de/DE/ ZahlenFakten/GesellschaftStaat/Bevoelkerung/Bevoelkerungsstand/Aktuell. html]. Accessed 1 Feb 2016.

19. Gesundheitsberichterstattung des Bundes: Todesursachenstatistik Methodik [https://www.gbe-bund.de/gbe10/abrechnung.prc_abr_test_ logon?p_uid=gast\&p_aid=0\&p_knoten=FID\&p_sprache=D\&p_ suchstring=2458::Todesursachenstatistik\%20Statistik\%20der\%20Gestorbenen \%20Todesursache\%20Sterbef\%E4lle]. Accessed 1 Feb 2016.

20. Pivodic L, Pardon K, Morin L, Addington-Hall J, Miccinesi G, CardenasTuranzas M, Onwuteaka-Philipsen B, Naylor W, Ruiz Ramos M, Van den Block, Lieve, Wilson DM, Loucka M, Csikos A, Rhee YJ, Teno J, Deliens L, Houttekier D, Cohen J. Place of death in the population dying from diseases indicative of palliative care need: A cross-national population-level study in 14 countries. J Epidemiol Commun Health. 2015;70:17-24.

21. WHO: Mortality Database [http://apps.who.int/healthinfo/statistics/mortality/ whodpms/]. Accessed 1 Feb 2016. 
22. Statista: Median age of the population in selected countries in $2014^{*}$ (in years) [http://www.statista.com/statistics/264727/median-age-of-thepopulation-in-selected-countries/]. Accessed 1 Feb 2016.

23. Pinzon E, Carlos L, Claus M, Zepf Kl, Letzel S, Fischbeck S, Weber M. Preference for place of death in Germany. J Palliat Med. 2011;14:1097-103.

24. Gaertner J, Weingärtner V, Wolf J, Voltz R. Early palliative care for patients with advanced cancer: How to make it work? Curr Opin Oncol. 2013;25:342-52.

25. Bruera E, Hui D. Integrating supportive and palliative care in the trajectory of cancer: establishing goals and models of care. J Clin Oncol. 2010;28:4013-7.

26. Gunjur A. Early in-patient palliative care consultation saves costs. Lancet Oncol. 2015;16:e321.

27. Stiel S, Pulst K, Krumm N, Ostgathe C, Nauck N, Lindena G, Radbruch L. Palliativmedizin im Spiegel der Zeit - Ein Vergleich der Ergebnisse der Hospiz- und Palliativerhebungen von 2004 und 2009. Palliativmedizin. 2010; 11:78-84.

28. McNamara B, Rosenwax LK, Holman C, D'Arcy J. A method for defining and estimating the palliative care population. J Pain Symptom Manage. 2006;32:5-12.

29. Traue DC, Ross JR. Palliative care in non-malignant diseases. J R Soc Med. 2005:98:503-6.

30. Mcllfatrick S. Assessing palliative care needs: views of patients, informal carers and healthcare professionals. J Adv Nurs. 2007;57:77-86.

31. Gómez-Batiste X, Martínez-Muñoz M, Blay C, Espinosa J, Contel JC, Ledesma A. Identifying needs and improving palliative care of chronically ill patients: a community-oriented, population-based, public-health approach. Curr Opin Support Palliat Care. 2012;6:371-8.

32. Simon ST, Gomes B, Koeskeroglu P, Higginson IJ, Bausewein C. Population, mortality and place of death in Germany (1950-2050) - Implications for end-of-life care in the future. Public Health. 2012;126:937-46.

\section{Submit your next manuscript to BioMed Central and we will help you at every step:}

- We accept pre-submission inquiries

- Our selector tool helps you to find the most relevant journal

- We provide round the clock customer support

- Convenient online submission

- Thorough peer review

- Inclusion in PubMed and all major indexing services

- Maximum visibility for your research

Submit your manuscript at www.biomedcentral.com/submit

C Biomed Central 